\title{
IMPLEMENTATION OF A PHYSICS-BASED DECISION-MAKING FRAMEWORK FOR EVALUATION OF THE MULTI- DISCIPLINARY AIRCRAFT UNCERTAINTY
}

\author{
Simon I. Briceno \\ Graduate Research Assistant, Aerospace Systems Design Laboratory (ASDL), Georgia Tech \\ Dimitri N. Mavris \\ Boeing Professor for Advanced Aerospace Systems Analysis, School of Aerospace Engineering, Director, ASDL
}

Copyright $@ 2003$ SAE International

\begin{abstract}
In today's business climate, aerospace companies are more than ever in need of rational methods and techniques that provide insights as to the best strategies which may be pursued for increased profitability and risk mitigation. However, the use of subjective, anecdotal decision-making remains prevalent due to the absence of analytical methods capable of capturing and forecasting future needs. Negotiations between airframe and engine manufacturers could benefit greatly from a structured environment that facilitates efficient, rational, decision-making. Creation of such an environment can be developed through a parametric physics-based, stochastic formulation that uses Response Surface Equations as meta-models to expedite the process. This paper describes the implementation of such an approach in order to demonstrate the types of insights that might be gained as an engine manufacturer tries to forecast the effects of the associated airframe uncertainties (structural, aerodynamic, etc. design changes) on engine related characteristics for the design of a hypothetical regional business jet.
\end{abstract}

\section{INTRODUCTION}

In the present business climate of Wall Street quarterly profit expectations, product launch decisions in the aerospace industry can no longer be based on engineering considerations alone. Current management processes could greatly benefit by the presence of a parametric tool that adequately describes and assesses new projects and opportunities based on both engineering and management considerations in an integrated environment.
In addition, a company cannot afford to base its decisions on information that does not account for the inherent uncertainty associated with engineering assumptions and customer requirements evolution. The inclusion of financial and competitive factors also is critical to decision-making. A new paradigm shift is therefore needed in the industry to facilitate these types of trade-offs. Although, this discussion may be pertinent to a variety of business settings, the authors have selected to study and understand the complex, at times non-cooperative, customersupplier relationship. More specifically, an airframe and an engine manufacturer were chosen as the representative customer and supplier, respectively, in order to formulate and rationalize the elements needed for the development of one such environment.

For this hypothetical scenario the engine manufacturer is assumed to be eager to capitalize on the opportunity of becoming the first supplier to successfully design, build and certify a competitive engine. This is particularly important in this context as airlines commonly expect their aircraft to be certified with products from all competing engine companies. Since this first certification could ultimately lead to early entry into the market prior to their competitor and potential profits, engine manufacturers are often willing to make concessions to ensure this competitive edge. Therefore, it is the authors' belief that engine manufacturers would especially benefit from the existence of a capability that allows management to provide some rationale and certainty in their decision-making process. The creation of such a framework involves defining key issues at the outset of the process. Initial steps use engineering analysis to provide a sense of cost and product uncertainty for management to consider. 
Customer requirements must be translated into engineering terms, and the corresponding uncertainty must be quantified. The second part of this paper focuses on the financial contribution to the decision-making tasks.

This paper constructs a representative environment around a notional commercial regional aircraft to illustrate the usefulness of this type of framework. The subsequent steps in the process involve utilizing current business, engineering and probabilistic tools to address the competitive nature of the problem and mitigate the inherent uncertainties. Formulations based on the by now well established fields of Game Theory and Real Options are employed to facilitate the decision-making process through a rapid and transparent business payoff analysis.

\section{CURRENT DESIGN PROCESS}

When an airframe manufacturer (customer) initiates the design process for a new product, engine manufacturers must quickly decide how to respond to the emerging needs of the vehicle. The time available to act varies according to the stage of the design process. The engine company (vendor) must decide if the project is worthwhile to pursue and generally employs some type of economic analysis based on return of investment, net present value or cost-benefit ratio. New aircraft also have a higher potential for fierce competition since no company may have a distinct advantage in producing the specialized engine needed. Decision makers deal with these issues by evaluating these assessment metrics based on personal insights or expert opinion usually in the absence of any in depth analytical assessment. Decisions are usually based on the ability to meet a set of requirements that is provided to them by the customer, while ensuring a reasonable return on investment. At this point the vendor will have to decide whether to accept or push back on some of these requirements. In many cases, the vendor feels pressured to secure the contract due to competition and to offer assurances and guarantees on the over-specified requirements that sometimes cannot be met simultaneously. However, uncertainty exists in the design of the vehicle and engine as both progress through the development. As a result, increased development costs or terminations of the contract occur if initial guarantees are not met. To avoid such incidents from occurring, an approach is proposed here that utilizes the engineering aspects of the problem to create a parametric trade-off environment which facilitates the use of probabilistic methods for the quantification and propagation of uncertainty throughout the analyses. Furthermore, since the market forces induce significant variability into the system requirements, one can argue that a robust solution is required with regards to a continuously evolving set of requirements.

\section{PROPOSED PROCESS}

In light of the considerations outlined above, a process is needed to assess the uncertainty associated with the development of a new aircraft. An effective decision-making technique relies on the ability of the engine manufacturer to quantify the uncertainty associated with the set of requirements given to them and determining an optimum strategy which mitigates the risk implied.

The possible management options must be clarified. Since engine design is a process, the development of a new engine is rarely characterized as simply a 'go' or 'no-go' decision. Alternatives to such oversimplification would include deferring a decision, continuing with initial development and then reassessing the project, and other such mixed management options. Finally, better means are required for dealing with the highly competitive nature of the aircraft engine market.

These methods may be characterized as essential "enablers" for this proposed decision support system. These enablers, critical for the development of the process shown above, include:

i) The creation of an integrated, physics-based modeling and simulation environment.

ii) The use of Design of Experiments DoEs and Response Surface Methods RSMs to expedite the trade-off analysis for transparency and visualization.

iii) Use of Joint Probability techniques to quantify the n-dimensional uncertainty impact.

iv) The concept of Robustness as an expected utility overall evaluation criterion. (Defined with regards to the associated uncertainty of evolutionary requirements.)

v) Game Theory to address the presence of multiple competitors.

vi) Advances in Real Options theory to address the inherent uncertainty associated with forecasted future market changes. 


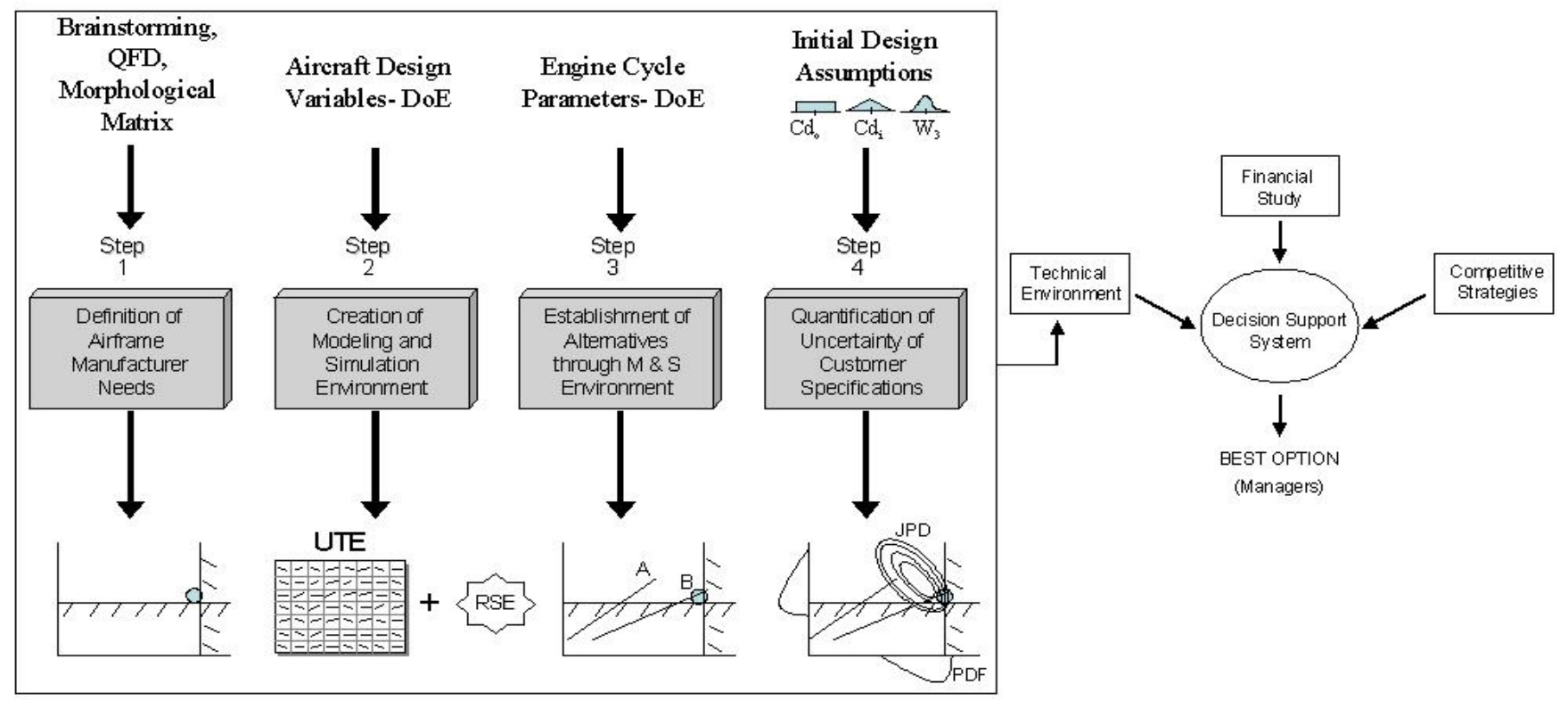

Figure 1: Proposed Process for Engine Development Decision Support System

The first part of this paper focuses on the fourth step of this process (Figure 1) by using a commercial jet, similar to a Boeing 737 next generation-class aircraft with a high-bypass turbofan engine, as a case study. The results from this case study, in which the reader can observe the application of the developing method from Ref. 1, are presented here along with a description of the on going financial study. For clarification purposes, a brief account of each step is now presented.

\section{STEP 1: ESTABLISHMENT OF CUSTOMER REQUIREMENTS}

Almost every process starts by defining and analyzing a set of stated requirements. This is where the voice of the customer is translated into terms and notions that the design team can comprehend. Specific metrics and targets are established to create a multidimensional design space constrained by various physical limits, as well as by customer requirements. This space is composed of engine characteristics such as thrust, engine weight, specific fuel consumption, emissions, noise, cost, reliability, maintainability, etc., or other airframe parameters such as block fuel weight. An engine's ability to meet any targets can then be modeled with a physics-based engine design routine, which is an essential step if a new engine design is to be developed. The final step in depicting the design space and defining the customer requirements in terms of engineering characteristics is to recognize where the constraints lie. This way, settings for metrics such as engine weight, thrust, or fuel consumption are identified to ensure satisfactory aircraft performance. Figure 2 illustrates two such notional engine architectures. The sets of vertical lines represent specific engines and their range of thrust ratings.

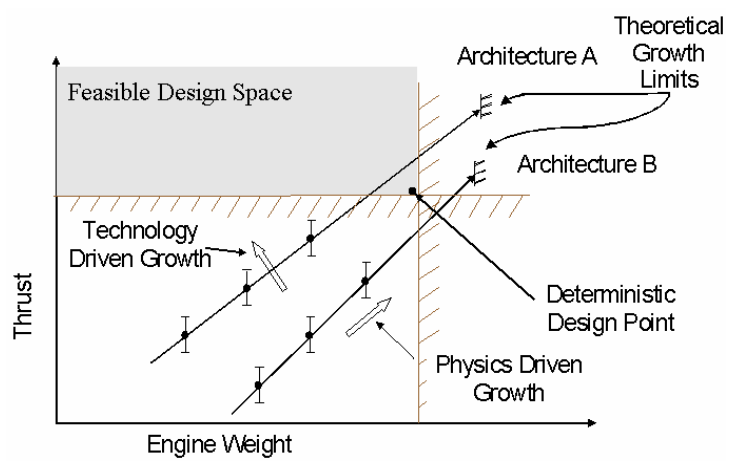

Figure 2: Relationship between Existing Architectures and New Requirements

The two notional architectures represent two engine "cores" to which changes can be made to create derivative engines. Although this is an $\mathrm{n}$-dimensional design space, two dimensions have been selected and shown here for visualization purposes. Similarly, other combinations can also be selected and presented. The design space in Figure 2 is shown in terms of engine thrust and engine weight and illustrates the two types of engine sizing trends that currently exist. The first growth trend is physicsdriven, by which an engine can be made to produce more thrust at the expense of increased weight. This 
is done by adding fan stages or by varying pressure or bypass ratios. The other growth trend is technology-driven. In this type of growth, thrust may be added to the engine at a constant weight, or even with a weight savings, by the use of new technologies and the expense of engine cost. It is possible to see that none of the six existing engines in the two architectures shown satisfy the requirements; thus, a newly developed engine design will be required. The design process might involve the modification of an existing engine in architecture A, or if this is not possible, the creation of a new engine. The question of which path is best now arises and represents the engineering view of the engine market problem. The following vehicle information represents the basis for the hypothetical case study in which the airframe and the engine manufacturer negotiate a new contract.

Hypothetical scenario: A final user, such as an airline, has requested the development of a new commercial jet. This vehicle must carry up to 150 passengers in two different classes at a low cost. The mission requirements are: a design range of 3,000 nautical miles, a cruise speed of Mach 0.82 , a takeoff and landing distance of no more than 6,500 feet. In addition, regulations cannot be violated for certification purposes, including federal aviation laws pertaining to aircraft certification. Table 1 identifies three metrics and their associated constraint values that the airframe manufacturer establishes as requirements to be met by the engine producer.

\section{Table 1: Constraint Values}

\begin{tabular}{lc}
\hline Constraint Metric & Value \\
\hline Min. Engine Thrust (lbs) & 32,300 \\
Max. Engine Weight (lbs) & 9,000 \\
Max. Fuel Flow (lbs/hr) & 8,385 \\
\hline \hline
\end{tabular}

The results presented here are only for illustration purposes of this proof of concept. The method can then be extended to a full-scale multi-dimensional representation.

\section{STEP 2: CREATION OF A PHYSICS-BASED MODELING AND SIMULATION (M\&S) ENVIRONMENT}

The modeling environment is developed by implementing current systems design methods as enablers to this process. An integrated modeling and simulation environment is initially created by means of physics-based routines or perhaps trusted semiempirical legacy codes. Within the design space, engineering parameters can be varied in order to meet the design constraints as shown in Figure 2. In this way, it will be possible to determine if an engine architecture can meet the constraints and to design an engine within the feasible region of the design space.

In this case study, once the design point has been identified by using the constraints, different architectures are developed to satisfy the feasibility of the design using available technology. These architectures are modeled by means of a parametric variation of cycle parameters in a physics-based engine analysis. The process begins by selecting the most influential cycle design variables. Employment of a Response Surface Methodology (RSM) allows for the creation of meta-models that will substitute the simulation codes. Meta-models, such as polynomial based Response Surface Equations (RSEs) can drastically decrease the computational time while maintaining a high level of accuracy. This is an essential tool in the creation of a rapid trade-off analysis framework [Ref 2].

Families of different engine architectures are then created after executing the analysis codes using the combinations of variables defined by a Design of Experiments (DoE). The codes may be linked sequentially as illustrated in Figure 3 . There are two independent paths, a propulsion path and an airframe related path. If the $M$ \& $S$ environment is used to generate engine architectures, the airframe configuration is held fixed and the engine cycle is parametrically varied using the engine design codes. This environment can also be used as a means to simulate uncertainty in the aircraft design. This analysis is performed using aircraft analysis routines.

The propulsion system analysis is based on the NASA Engine Performance Program (NEPP) and the Weight Analysis of Turbine Engines (WATE) code. NEPP is a NASA/industry developed cycle analysis program that determines the onedimensional, aerodynamic and thermodynamic characteristics of the flow going through the engine [Ref. 3]. Subsequently, information generated in NEPP is fed into WATE which performs a flow-path analysis and determines the engine weight and other physical characteristics [Ref. 4].

The aircraft design code used for the performance uncertainty analysis is NASA's FLight OPtimization System (FLOPS). This is an aircraft synthesis and sizing program that is capable of generating aircraft designs with a given range, passenger capacity and 
aircraft geometry and determines if a feasible solution exists [Ref. 5].

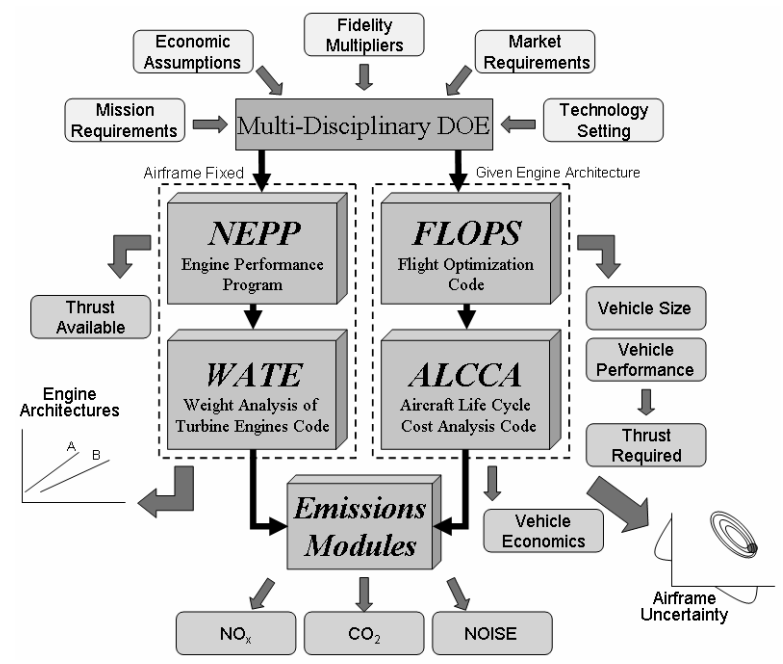

Figure 3: Analysis Code Execution Process

This multi-disciplinary design code is mainly used to show the effects of varying aircraft components weight and aerodynamic changes on required engine thrust, weight, fuel-flow, and engine specification metrics. The aircraft economics are determined through an Aircraft Life Cycle Cost Analysis code (ALCCA).

As such, the engine manufacturer will have to design for this requirement in order to account for the effects of airframe uncertainty.

Table 2 and 3 list the ranges for the engine cycle and aircraft uncertainty variables used in this case study in order to construct the DoE. There are however, additional disciplines besides propulsion, aerodynamics and structures that are included in the same DoE. Such as factors associated with mission requirements, economic assumptions, fidelity multipliers, market requirements, and technology settings that account for other aircraft design related variations. Table 3 is included in the DoE to model the aircraft uncertainty due to aerodynamic and structural changes. The methods used to quantify this uncertainty are described in step 4 of this paper.

Results of the parametric variations shown in Table 3 identify quantities such as the maximum engine weight required to satisfy the required aircraft configuration and performance objectives.

As such, the engine manufacturer will have to design for this requirement in order to account for the effects of airframe uncertainty.
Table 2: Engine Cycle Variables and Ranges

\begin{tabular}{lc|c|c}
\hline Design Variable & Min & Nominal & Max \\
\hline Fan Pressure Ratio & & & \\
(FPR) & 1.5 & 1.65 & 1.8 \\
Extraction Ratio (ER) & 1.05 & 1.2 & 1.35 \\
Turb. Inlet Temp (T4) R & 3050 & 3150 & 3250 \\
Mass flow rate (lbm/sec) & 600 & 700 & 800 \\
$\begin{array}{l}\text { Fan Efficiency (FEF) } \\
\text { High Pressure }\end{array}$ & 0.88 & 0.885 & 0.89 \\
$\begin{array}{l}\text { Comp. Eff. (CEF) } \\
\text { Overall Pressure }\end{array}$ & 0.87 & 0.875 & 0.88 \\
Ratio (OPR) & 30 & 32.5 & 35 \\
\hline \hline
\end{tabular}

This part of the DoE has a fixed engine configuration. This integrated $\mathrm{M}+\mathrm{S}$ environment leads to the creation of what will be referred to here as a Unified Trade-off Environment or UTE. This environment was first proposed as a concept in [Ref 6.]

\section{Table 3: Aircraft Aerodynamic and Structural Uncertainty Variables}

\begin{tabular}{|c|c|c|c|}
\hline Design Variable & Min & Nominal & Max \\
\hline $\begin{array}{l}\text { Zero-Lift Drag Coef. } \\
\text { Multiplier }\end{array}$ & 0.9 & 1 & 1.1 \\
\hline $\begin{array}{l}\text { Induced Drag Coef. } \\
\text { Multiplier }\end{array}$ & & 1 & 11 \\
\hline $\begin{array}{l}\text { Multiplier } \\
\text { Fuselage Weight }\end{array}$ & 0.9 & 1 & 1.1 \\
\hline Multiplier & 0.99 & 1.01 & 1.03 \\
\hline $\begin{array}{l}\text { Wing Weight Multiplier } \\
\text { Horiz. Tail Weight }\end{array}$ & 0.99 & 1.01 & 1.03 \\
\hline $\begin{array}{l}\text { Multiplier } \\
\text { Vertical Tail Weight }\end{array}$ & 0.99 & 1.01 & 1.03 \\
\hline $\begin{array}{l}\text { Multiplier } \\
\text { Mertigal Weigt }\end{array}$ & 0.99 & 1.01 & 1.03 \\
\hline
\end{tabular}

The utilization of a DoE approach explores the concept of RSEs to parametrically change aircraft requirements, engine or aircraft design parameters, and assumptions made on the aircraft design and engine efficiencies, thus simplifying the process to facilitate a rapid trade-off environment. From this type of environment it is possible to extract information about aircraft constraints and available engine performance simultaneously.

A Unified Tradeoff Environment (UTE) is developed using multiple system-level DoE's as defined above. This trade-off environment also enables rapid what-if exercises and investigations by instantaneously showing the effects of the input variables into the outputs and finally as a debugger by spotting any potential modeling error. The reader is referred to Reference 1 for a detailed UTE description. 


\section{STEP 3: ESTABLISHMENT OF ALTERNATIVES}

One advantage of this simulation environment is its capability to create engine architectures. These are represented by changes in two main engine characteristics such as thrust and weight while keeping the same engine architecture. Engine performance data is then plotted according to customer needs, enabling engine designers to spot new designs and also compare existing available architectures with these needs.

A different family within each architecture is generated by changing the cycle parameters of the engine core while keeping a fixed fan diameter. Three engine architectures are presented for this hypothetical scenario using this simulation analysis.

\section{STEP 4: QUANTIFICATION OF UNCERTAINTY OF CUSTOMER SPECIFICATIONS}

Customer requirements are specified as deterministic values that translate into constraints on the engine design space. However, these requirements rarely stay the same throughout the entire design process. These engine specifications are ultimately a function of aerodynamics, structures and other aircraft characteristics that will change whenever the aircraft design changes.

Mission uncertainty faced by the customer results in aircraft performance changes, yielding further variation of engine specifications. The vendor observes this variation of engine requirements as a migration of the engine design point. An illustration of this migration scenario is shown in Figure 4. Once the Modeling and Simulation Environment has been created, it is possible to challenge the required solution and possible deviations from the original needs. For instance, the solution point contains assumptions about:

i) Structures: If for any reason the initial design empty weight of the vehicle cannot be met when performance and takeoff gross weight are fixed, the engine manufacturer might be asked to lower the engine weight and or fuel consumption to help in reducing the overall vehicle empty weight or block fuel weight. Since there is usually a competing engine manufacturer waiting for an opportunity to enter the game, the company that is willing/capable of providing such a reduction might be the one chosen to power the vehicle. This is illustrated in Figure 4 where the maximum allowed engine weight is decreased, shifting the engine design point to (1).

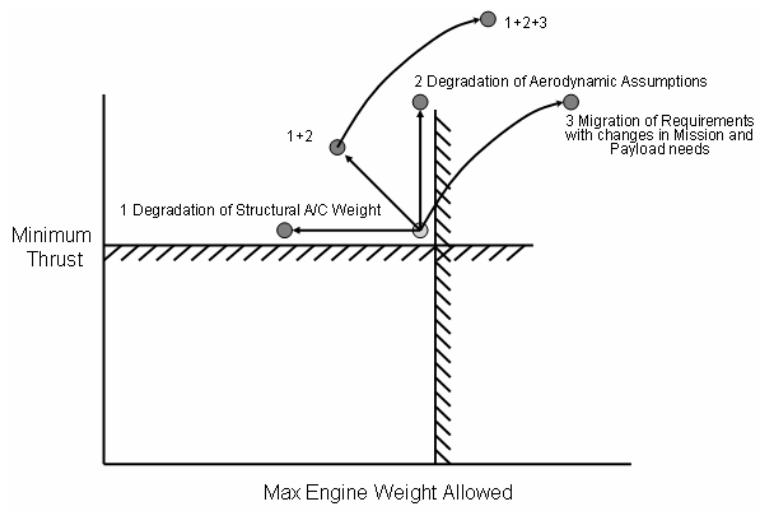

Figure 4: Migration of the Design Point with Uncertainty

ii) Aerodynamics: uncertainty associated with the vehicle aerodynamics may lead to a degradation of the aerodynamic drag coefficients and properties which may increase the needed thrust to maintain the target cruise speed. This situation results in a displacement of the design point to location (2) as shown above.

iii) Mission Evolution: Initial user needs usually change over time. These changes include variations in payload and range that regularly occur with time as new versions of this vehicle will be introduced on the market. This migration, due to growth needs, is denoted as (3).

iv) Combinations: As shown in the figure, it is possible to observe that combinations of these three scenarios can possibly change the design point.

Uncertainty in engine specifications is modeled by introducing variations in the assumptions made by the customer. For instance, the airframe manufacturer requires that the performance remain unchanged with a reduction in engine weight. As the aircraft design matures, the empty weight estimation at the outset is no longer valid. The customer needs to maintain range, payload, and performance. To balance this increase in weight, the airframe manufacturer may pass some of the weight reduction responsibility to the engine manufacturer, requiring a reduction in engine weight to meet the user performance needs. At this point, the engine manufacturer may no longer guarantee meeting the initial design requirements with full confidence. The process for implementing such analysis on the uncertainty of the provided engine requirements is illustrated in Figure 5. [Ref. 7,8]. The representation of the modeling and simulation environment, via 
polynomials as expressed by the created RSEs, greatly facilitates the use of a Monte Carlo Simulation approach to obtain the probabilistic forecasts.

Monte Carlo Simulation

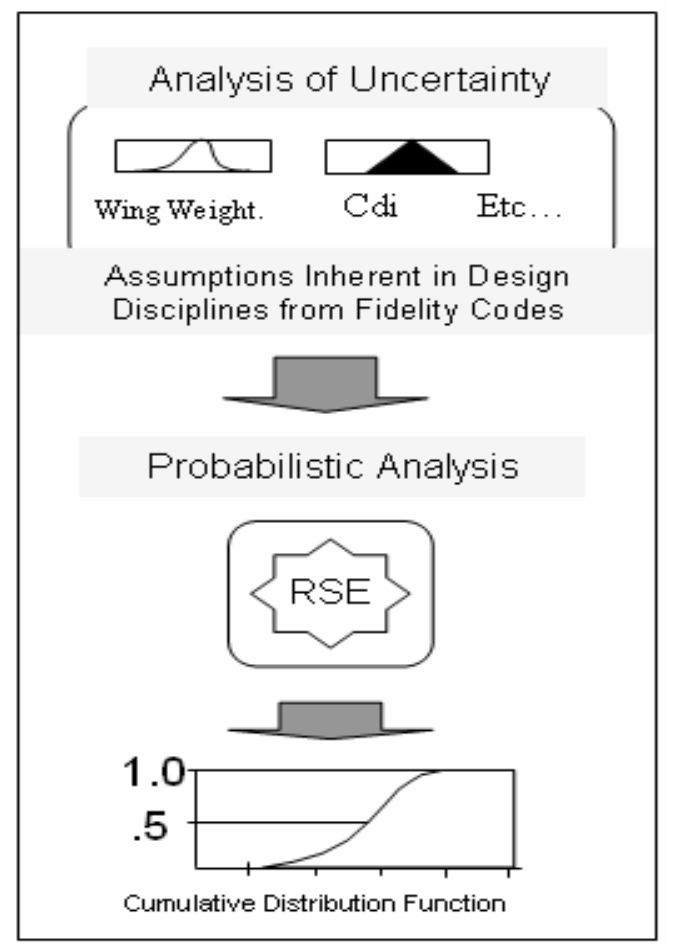

Figure 5: Probabilistic Analysis Process

A probability distribution of an appropriate shape of is placed over the ranges set for each parameter. These distribution shapes can be normal, triangular, beta, or any other shape that best represents the perceived confidence and represent the probability of change of any given design metric (aerodynamic, structural, etc.) As the design development matures and the confidence associated with these estimates increases (i.e. reduction in the variability range) these shape functions will also be modified to reflect the change. Since the shape functions are time dependent this formulation may be characterized as a stochastic one.

The distributions defined are then provided as inputs into the computationally expedient response surface polynomial equations, and a Monte Carlo Simulation is performed. As the conclusion of this iterative process probability distribution functions (PDFs) or cumulative distribution functions (CDFs) may be obtained for each of the responses of interest. Once these distributions have been evaluated for each engine response, or metric of interest, and their corresponding correlation coefficients " $\rho$ " have been determined (using the DoE obtained from observed results), joint probability curves may be introduced by superimposing two or more PDFs into the engine design space. The same modeling and simulation techniques used in creating engine architectures are used to model aircraft uncertainty.

The process, as illustrated in Figure 5, models the data from FLOPS in the form of response surface equations and runs a Monte Carlo Simulation to create a statistical representation of the uncertainty. Each variable in the uncertainty study is given an assumed range interval depending on the level of knowledge of that variable. This study is carried out with first order methods in the preliminary design phases.

In the first iteration of this process it was assumed that the PDF of the customer needs could be represented by a normal distribution. In order to visualize the needs on engine specifications in a multidimensional space, it is necessary to create a Joint Probability Distribution (JPD). This has been done for thrust and weight parameters, by using the equation 1 , where " $f$ " is the frequency of the JPD, " $x$ " and " $y$ " are any parametric axes such as thrust and weight and "a" and " $b$ " are specific values of $x$ and $y$ respectively. The JPD equation uses the normal distribution parameters, with mean $(\mu)$, standard deviation $(\sigma)$, and correlation coefficients $(\rho)$ of pairs of criteria. For illustration purposes a partial sample data set was obtained for the examined hypothetical case study and is presented in Table 4.

Table 4: Distribution Characteristics

\begin{tabular}{lllll}
\hline & $\mu$ & $\sigma$ & $\rho$ & $\begin{array}{c}\text { With } \\
\text { Variable }\end{array}$ \\
\hline Thrust & 32289 & 312.64 & ---- & --- \\
Weight & 8452 & 368 & -0.6 & Thrust \\
Fuel flow & 8229 & 2.47 & -0.7 & Thrust \\
\hline \hline
\end{tabular}

It is worth noticing here that correlation coefficients between thrust and engine weight and thrust and fuel flow are both either negative or inversely correlated. At first this appears to be counter intuitive, however; these relationships are correct. The correlations shown here are not based on the physics of the problem instead they are based on customer preferences and wants. 
Further insight may also be gained by super imposing the joint probability density contours obtained by the customer requirements uncertainty quantification analysis onto the physics-based attainable design architectures. One such mapping of this n-dimensional space has been captured and projected in two dimensions. Thrust against engine weight is illustrated in Figure 9.
Note that the frequency contours extend into the infeasible space, indicating that there is a small chance that the customer requirements could end up lower than what was originally negotiated. However, there is a high probability that the original requirements will shift slightly towards the feasible space.

$$
f_{X Y}(a, b)=\frac{1}{2 \pi \sigma_{X} \sigma_{Y} \sqrt{1-\rho_{X Y}{ }^{2}}} \exp \left\{\frac{1}{2 \rho_{X Y}{ }^{2}-2}\left[\left(\frac{a-\mu_{X}}{\sigma_{X}}\right)^{2}-2 \rho_{X Y}\left(\frac{a-\mu_{X}}{\sigma_{X}}\right)\left(\frac{b-\mu_{Y}}{\sigma_{Y}}\right)+\left(\frac{b-\mu_{X}}{\sigma_{X}}\right)^{2}\right]\right\}
$$

Engine Decision Environment

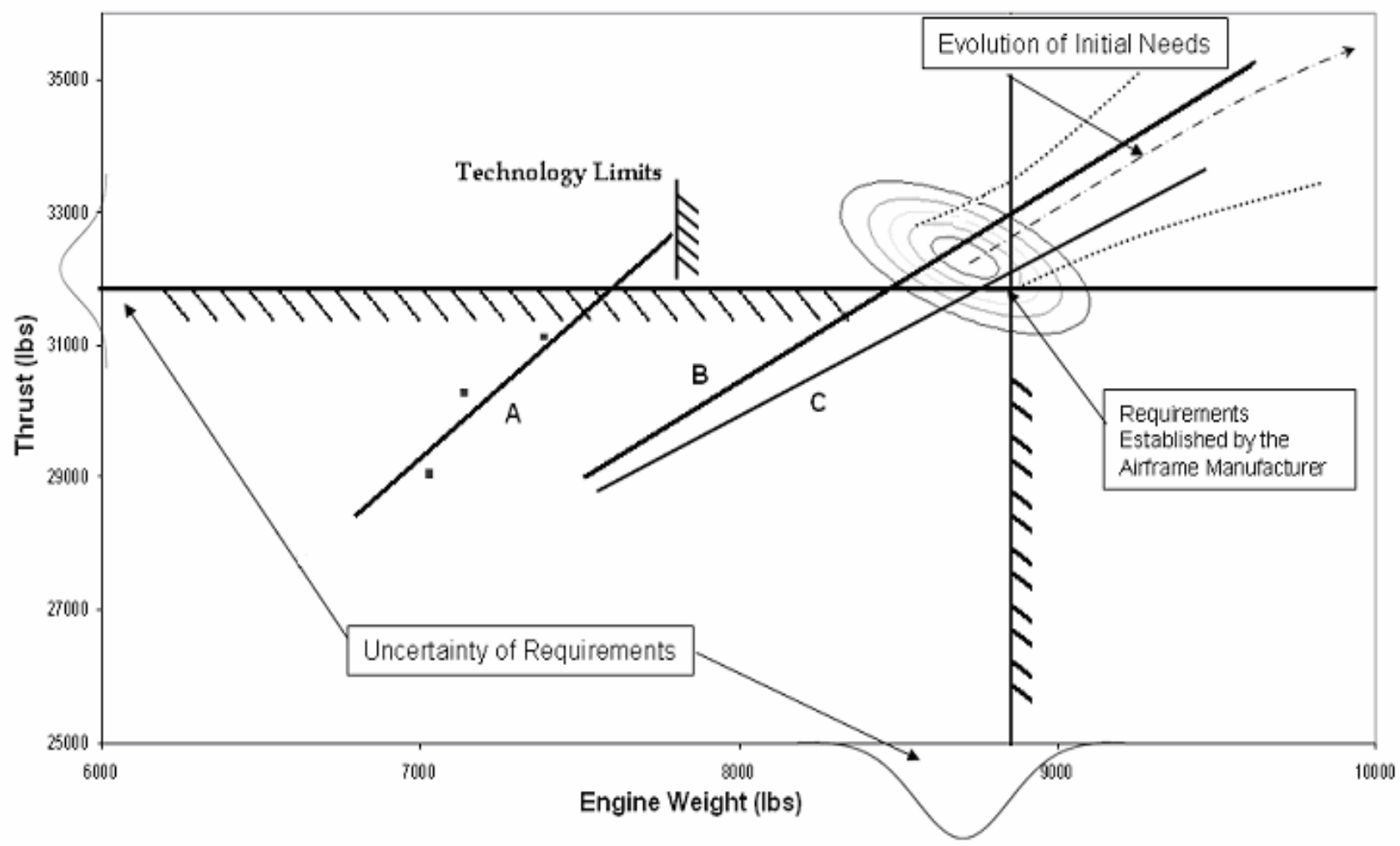

Figure 6: A Probabilistic View of the Engine Design Requirement Space

This situation will therefore reduce slightly the available design space. For this notional example, to meet the new requirements obtained from this shift of the design point with a high level of confidence, the final engine design must have slightly higher thrust with lower weight and/or lower fuel consumption than the original deterministic needs. It can be inferred therefore that architecture $B$ is probably the best alternative to consider in Figure 6, since it meets the current needs with higher probability of success and at the same time its physical growth line is extended above the forecasted line of evolutionary requirements. Architecture A, however, shows a larger increase of thrust with smaller weight penalties. This alternative can be generated by the infusion of technologies, but even though it appears superior to the other architectures, it still does not provide a robust solution due to technological limitations. A preliminary modeling of evolving aircraft requirements, depicted by the dashed line with positive slope that starts at the peak of the JPD, is carried out through a 
simple variation of aircraft range and passenger capacity. At this point, the environment provides enough information about the aircraft uncertainty so the engine manufacturer can make a preliminary assessment on future needs. It must be noted again that in a real scenario, this environment would be multidimensional, accounting for variability in all disciplines of aircraft design. Only the effects of aircraft weight and wing aerodynamics have been modeled here to show this notional scenario.

In addition to the probabilistic analysis that produces the environment shown in Figure 6, further studies can identify the probability of a specific architecture or a family of engines meeting the airframe design requirements. The concept of "probability of success" is employed using the notional scenario as a case study. To better understand this concept, Figure 7 illustrates two hypothetical engines that may exist on this environment.

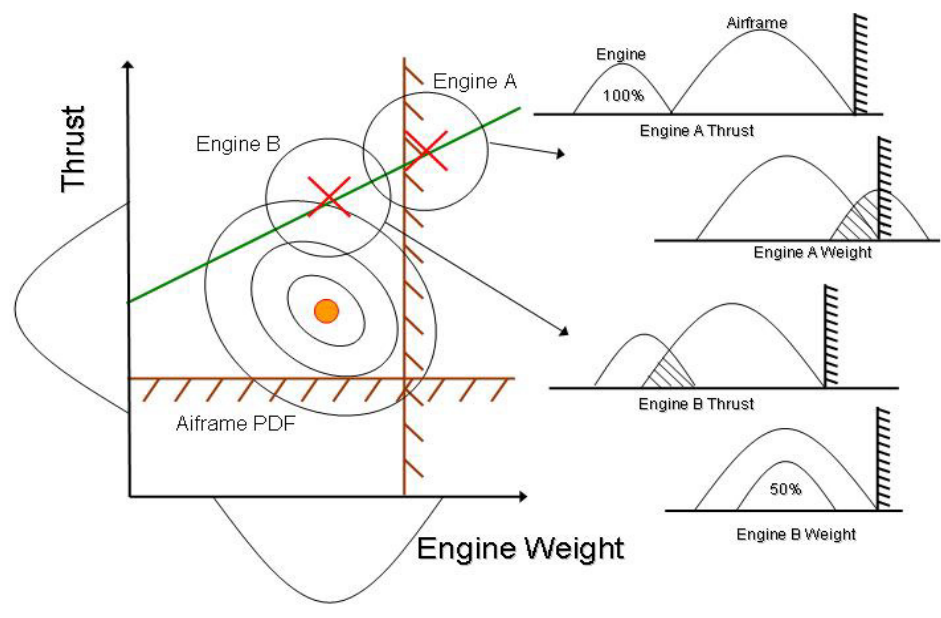

Figure 7: Hypothetical Probability of Successes

Two engines, $A$ and $B$, are possible examples of such an environment. These results indicate that for engine $A$, there is a $100 \%$ chance of meeting the thrust required and a $20 \%$ chance or less of meeting the required engine weight. Engine $B$ has a lower probability of meeting thrust, but a higher probability of meeting the weight requirement. The Crystal Ball software [Ref. 8] provides a good numerical estimation of these probabilities.

This type of analysis proves useful in determining an approximate likelihood that a specific engine will fall within the envelope of a design requirement. Trade-offs are performed by giving a specific metric of interest more importance. If two or more engines exist that potentially could satisfy the requirements, then a separate trade-off analysis between the engines can determine which engine has the highest chance of meeting such requirements. If these trade-offs yield no clear winner, further analysis in other dimensions could single out a winning engine in such competition.
Using the case study presented in this paper, the probability-of-success analyses are performed and the results are illustrated in Figure 8. A typical engine is generated (as described in step 3 ) for this thrust class and considered to be a good candidate for the airframe. Two more representative engines are overlaid onto the environment to observe real results using these techniques.

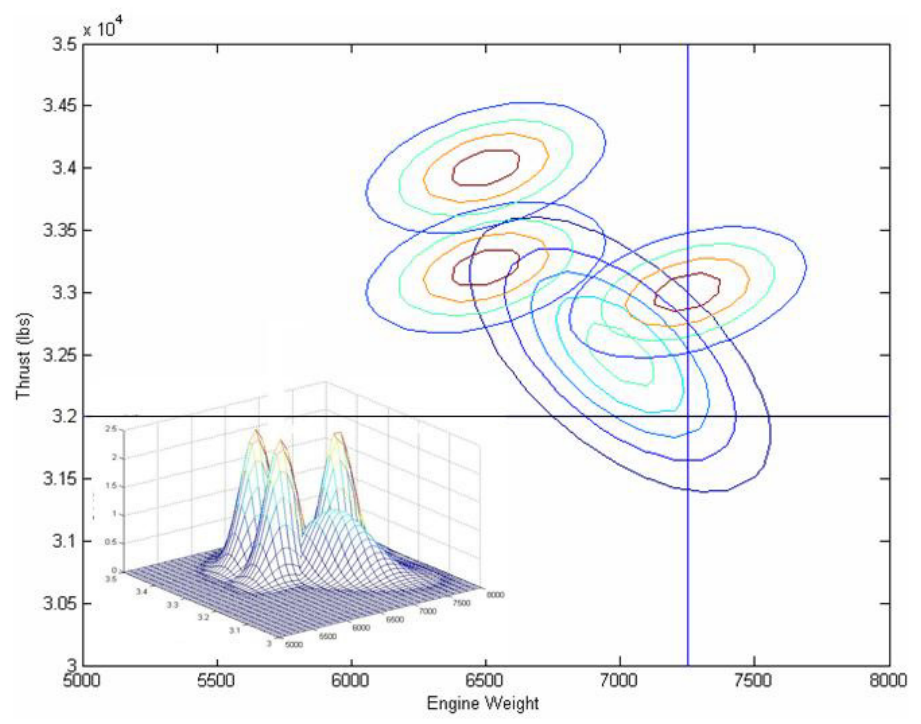

\section{Figure 8: Results of Joint probability Environment with three competitive engines}

At this point this design space has sufficient information to make a preliminary decision as to the feasibility of a particular engine. The "winning" engine(s) are then analyzed further by means of a thorough financial and competitive study in order to determine economic viability.

\section{FINANCIAL AND COMPETITIVE STUDIES}

A game theoretic approach to decision making can be employed to design and optimize these kinds of engines. In this case the decision maker has an expected utility that he is trying to maximize (for instance, the probability of fulfilling and exceeding all of the criteria posed) and nature is standing in his way, opposing and forcing him to accept a compromised solution (i.e. environmental constraints). This formulation is inadequate in the presence of any competition and is therefore in need of enhancement to accommodate this added complexity. Fortunately, a lot of advances have been made over the past few years in the field of decision making and new innovative approaches and algorithms have been proposed in the field of game theory. An approach using game theory thus will provide a mathematical basis for: enumerating decisions available, evaluating options or "moves", ruling out moves that do not make strategic sense, determining whether partnerships are optimal strategies, and conducting "what-if" analysis for various scenarios. In fact, the more complicated and competitive a given market is, the more there is a need for 
quantitative methods for analyzing business strategy and the influence of engineering decisions on that strategy. It is therefore suitable to borrow some of these algorithms, couple them with the physics of the problem and exercise them to guide the decision-making process.

Figure 9 shows two possible alternatives to evaluate. Notice that there are some developed engines in architectures $\mathrm{B}$ and $\mathrm{C}$.

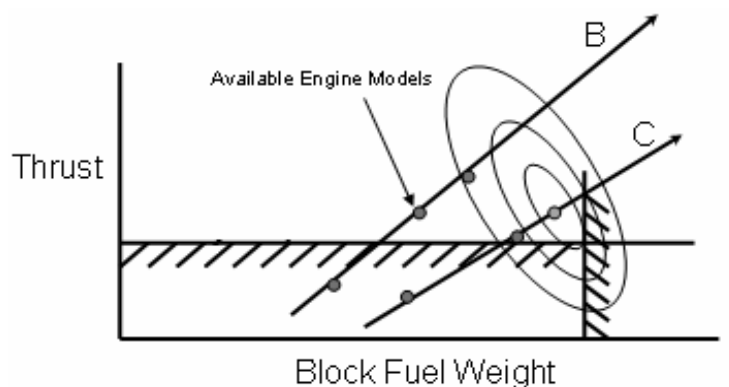

Figure 9: Mapping of available alternatives upon the JPD requirement space

Having determined the engineering confidence for a new engine at the present time, it is necessary to predict a confidence interval for the future requirements and costs. Rather than using net present value, tools such as real options may be used. The concept of real options [Ref. 9] is a real management and project assessment tool that allows managers to forecast the present value of a project based on market conditions similarly to the analysis of stock options. Its objective is to better characterize the decision-making alternatives in a project.

If for instance, two engine alternatives satisfy the technical environment, they are said to be feasible engines. Even though all performance requirements have been met, managers must now determine which will become the most economically viable engine of the two. Now, a completely different study, based on the lifecycle cost of the engine allows the manager to address all the non-technical related factors that affect the viability of an engine alternative.

There is a host of different market and social factors that contribute to the viability of an engine. From the two available engines, although both meet the technical/performance requirements, one of them will be cheaper to produce than the other. The engine manufacturer may have to invest resources in order to produce the other engine. Such investment may include the establishment of a new manufacturing plant with new tools and machinery. New suppliers may also have to be acquired. There may also be a negative or positive impact on the social environment. Managers are therefore more inclined to choose the cheaper engine to produce. In addition, they are also concerned with the adaptability of the engine. If the engine can be modified slightly to service other aircraft variants, the customer will be more likely to buy that engine. An engine that is also similar to other existing engines will facilitate its maintainability and serviceability. Such a common platform will also require less training in its production since there is a built-in learning curve already created from other similar engines. A major factor associated with the choice of an upgradeable engine over that of a new engine is that of risk and competition. The risk of losing a contract could be more significant than many of the factors suggested above. Political motivations may also impact the decision-making process.

The real options tool provides managers with a portfolio of options that may include but are not limited to the following actions: abandon, wait, contract, expand or switch options. Initially, a qualitative management screening process may generate iso-cost lines that can be overlaid onto the technical environment. This will generate lines of equal cost for different engines. A base case NPV analysis determines what the project will entail, what assets are available, what initiatives have been drawn, what initial strategies have been established, etc. A discounted cash-flow and time series forecasting analysis is performed for each qualified project (engine). A Monte Carlo Simulation is run over all these possible combinations where the inputs are the revenues, costs, tax rates, discount rates, capital expenditures, depreciation, etc. and the output is the net present value. The real options problem framing is a visual tree diagram of the various projects and paths available to choose from. At each node, a strategic option (expand, contract, abandon, etc.) can be utilized. An optional step involves the optimization of the portfolio and resources. Results are then reported as feedback to the initial steps and the process iteratively finds an optimum engine. Figure 10 summarizes the above iteration process.

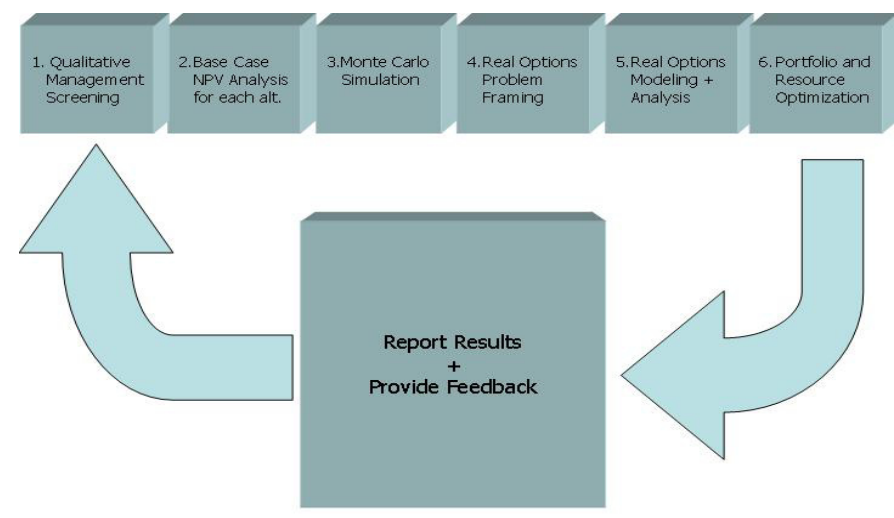

Figure 10: Steps in Real Options Analysis

By employing this type of process, managers equip themselves with more knowledge about the life-cycle cost of each individual engine. In addition to the cost of the production, managers must account for and respond to other entities that are competing for the same contract. 
Finally, managers must determine which engine is both feasible and viable. At this point of the implementation, algorithms are written that will enable the rapid and methodical assessment of all design options available in the linked business and engineering environment in search of the best possible alternative. Each potential candidate within this environment will be described as a vector of attributes comprised of performance and economic characteristics as well as their associated probabilities of occurrence.

One of the advantages of this integrated decision support system, as mentioned earlier in the paper, is the ability to integrate engineering, mathematical and business processes and decision tools. The accumulation of these diverse set of methods enables the decision makers to obtain valuable insights and allows them to base their selection on a multitude of potential evaluation criteria. For instance, the overall evaluation criterion may be based on the determination of a robust solution. In this context, a robust solution will be defined as a design that is relatively insensitive to requirement changes. A robust solution will therefore remain both feasible and viable in time with respect to any potential requirements evolution. Furthermore, Real Options theories introduced in the financial study are employed here which enables the assessment of future options which may or may not occur. This will make possible the forecasting of the best strategy considering future needs and value of the needed engineering research regarding the contract [Ref 10.]. This Real Options tool will then be combined with a Game Theoretic approach which will be linked to the engineering framework by the use of decision trees and matrices capable of analyzing all pertaining fields (i.e. engine characteristics, market conditions, etc.) in a rapid and accurate method. Finally, any one of the options presented above could be formulated as a multi attribute decision-making (MADM) problem and call for the most suitable of the 17 or so MADM techniques available in the literature to determine the best solution.

\section{CONCLUDING REMARKS}

Airframe and engine manufacturers' negotiations can benefit greatly from increased business effectiveness due to a structured decision-making environment. Creation of such an environment can be facilitated by a parametric physics-based, stochastic formulation that uses meta-models to expedite the process and allows for rapid trade-off and what-if exercises. The implementation of such an approach has been formulated and presented in this paper, demonstrating the potential insights gained by the engine manufacturer as he tries to forecast the effects of uncertainties, as well as future vehicle requirements, on engine related characteristics.
Furthermore, this method provides a decision support system which integrates technical and management concerns and provides rapid and valuable insights to decision makers as to what is the best option or strategy, today and in the near future, concerning the investment into a new engine development. The results provided by this hypothetical scenario and decision support system are useful examples that highlight the potential decision-making capabilities of this type of environment.

Finally, Game Theoretic and Real Options studies are currently being developed for this hypothetical scenario as potential means to quantify and evaluate the influence of present and future market competition on the decision making process.

\section{REFERENCES}

1. Mavris, D.N., Briceno, S.I., Fernandez, I., "Development of a Strategic Business Decision Making Environment for Commercial Jet Engine Selection" AIAA-2003-0720

2. Mavris, D.N., Olson, E.D., "Development of Response Surface Equations for High-Speed Civil Transport Takeoff and Landing Noise" SAE 975570

3. Klann J. L., Snyder C. A., "NASA Engine Performance Program" Aero propulsion Analysis Office, NASA Lewis Research Center, Cleveland Ohio, March 1997.

4. Boeing Military Airplane Development, NASA-Glenn Research Center, PSAO office "Weight Analysis of Turbine Engine" 1979.

5. McCullers L. A., "Flight Optimization System" NASA Langley Research Center, Hampton, VA, April 2001.

6. Mavris D. N., Birney, M. "Formulation of a Stochastic, Physics-Based Strategic Business Decision Making Environment" AIAA-2002-5850

7. Baker, A. P., "The Role of Mission Requirements, Vehicle Attributes, Technologies, and Uncertainties in Rotorcraft System Design ", Ph.D. Thesis, Georgia Institute of Technology, 2002.

8. Kirby, M.R., "A Method for Technology Identification, Evaluation and Selection in Conceptual and Preliminary Aircraft Design", Ph.D. Thesis, Georgia Institute of Technology, March 2001.

9. Copeland, T. E., Antikarov, V. "Real Options: A Practitioners Guide" Texere, New York. 2001.

10. Grenadier, S. "Game Choices: The Intersection of Real Options and Game Theory" Risk Books, London UK. 2000

\section{CONTACT}

Simón I. Briceño: simon.briceno@asdl.gatech.edu 